\title{
Embarazo a término en útero bicorne con cuerno rudimentario no comunicante. Reporte de un caso
}

\author{
Martha Rondon-Tapia , ${ }^{1}$ (i) Eduardo Reyna-Villasmil .2 (iD Duly Torres-Cepeda. ${ }^{3}$
}

\section{RESUMEN}

El útero bicorne con un cuerno rudimentario no comunicante puede estar asociado con complicaciones ginecológicas y obstétricas tales como infertilidad, endometriosis, hematómetra, anomalías del tracto urinario, abortos y partos pretérmino. La escisión del cuerno rudimentario debe hacerse fuera del embarazo, con seguimiento durante el embarazo, en busca de complicaciones. Se presenta el caso de una paciente de 40 años, que presentó dolor abdominal y sangrado vaginal, con un embarazo a término en un cuerno rudimentario no comunicante de un útero bicorne. El examen físico mostró distensión abdominal y pérdida del contorno uterino y no se palparon partes fetales. Se realizó el diagnóstico provisional de ruptura del útero. La laparotomía de emergencia reveló feto muerto y deformado, de 37 semanas, en útero bicorne con un cuerno rudimentario no comunicante roto, junto con placenta ácreta. Se realizó extracción del saco gestacional, feto y placenta e histerectomía subtotal con conservación del anexo izquierdo.

Palabras clave: Cuerno rudimentario no comunicante, Útero bicorne, Embarazo a término.

Term pregnancy in a bicornuate uterus with non-communicating rudimentary horn. Case report.

\section{SUMMARY}

The bicorne uterus with a rudimentary non-communicating horn may be associated with gynecological and obstetric complications such as infertility, endometriosis, hematometra, urinary tract abnormalities, abortions, and preterm deliveries. Excision of the rudimentary horn should be done outside of pregnancy, with followup during pregnancy, looking for complications. We present the case of a 40-year-old patient, who presented abdominal pain and vaginal bleeding, with a full-term pregnancy in a rudimentary non-communicating horn of a bicorne uterus. Physical examination showed abdominal distention and loss of uterine contour and no fetal parts were palpated. The provisional diagnosis of rupture of the uterus was made. Emergency laparotomy revealed a dead and deformed fetus, 37 weeks old, in a bicorne uterus with a broken rudimentary non-communicating horn, along with an acretic placenta. Extraction of the gestational sac, fetus and placenta and subtotal hysterectomy with conservation of the left annex were performed.

Keywords: Rudimentary horn not communicating, Bicornuate uterus, Term pregnancy.

${ }^{1}$ Médico especialista. ${ }^{2}$ Doctor en Medicina Clínica. Especialista en Ginecología y Obstetricia. ${ }^{3}$ Doctora en Ciencias Médicas. Especialista en Ginecología y Obstetricia. Servicio de Obstetricia y Ginecología. Hospital Central "Dr. Urquinaona”. Maracaibo. Estado Zulia. Correo de correspondencia: sippenbauch@gmail.com

Forma de citar este artículo: Rondon-Tapia M, Reyna-Villasmil E, TorresCepeda D. Embarazo a término en útero bicorne con cuerno rudimentario no comunicante. Reporte de un caso. Rev Obstet Ginecol Venez. 2021; 81(4):415-419. https://doi.org/10.51288/00810414

\section{INTRODUCCIÓN}

El embarazo dentro de un cuerno rudimentario de un útero bicorne es muy raro con una incidencia de 1 en $100000-140000$ embarazos (1,2). En cerca del $90 \%$ de los casos, la resultante obstétrica habitual es la ruptura uterina durante el segundo trimestre, mientras que solo $10 \%$ de los casos llega al término del embarazo. Esta condición es potencialmente fatal 


\section{EMBARAZO A TÉRMINO EN ÚTERO BICORNE CON CUERNO RUDIMENTARIO NO COMUNICANTE. REPORTE DE UN CASO}

debido a que el diagnóstico preoperatorio puede ser muy difícil (3-7). En la literatura nacional se encontró un caso, reportado en 2012, de una paciente de 18 años, con un embarazo de 30 semanas más dos días, con diagnóstico de útero bicorne y agenesia renal quien desarrolló preeclampsia y requirió interrupción del embarazo (6). Se presenta el caso de una paciente con un embarazo a término en útero bicorne con cuerno rudimentario no comunicante.

\section{HISTORIA CLÍNICA}

Se trata de una paciente de 40 años, VII gestas, V paras, I aborto, quien fue referida a la emergencia obstétrica por presentar dolor abdominal de moderada intensidad, durante los últimos 10 días, acompañado de sangrado genital en escasa cantidad en las últimas 24 horas. La paciente negaba la asistencia al control prenatal, al igual que antecedentes personales o familiares de importancia. Los 5 partos fueron por vía vaginal y espontáneos, sin complicaciones, atendidos en 3 hospitales diferentes.

Al examen físico, se observó evidencia de choque hipovolémico (marcada palidez cutáneo-mucosa, piel fría, frecuencia cardiaca 160 latidos por minuto, presión arterial 80/60 mm de $\mathrm{Hg}$ ). El abdomen estaba distendido, con dolor a la palpación superficial y profunda. No se palpaba el contorno uterino ni las partes fetales. La evaluación obstétrica no detectó la presencia de frecuencia cardiaca fetal y/o movimientos fetales Todos estos signos llevaron al diagnóstico presuntivo de rotura uterina.

Durante la laparotomía de emergencia se encontraron adherencias densas con el epiplón e intestino delgado sobre una tumoración compatible con saco gestacional flotante en la cavidad y sangre libre en cavidad abdominal (aproximadamente $2500 \mathrm{~mL}$ ). Al realizar la incisión sobre la tumoración, se observó un gran hematoma, de aproximadamente 6 x 10 centímetros, el

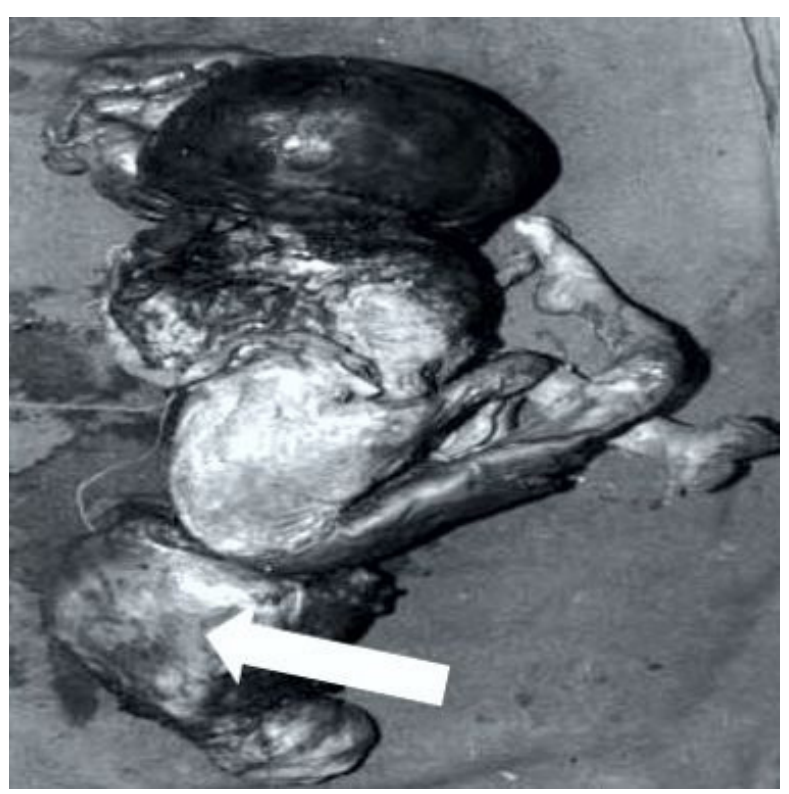

Figura 1. Feto muerto con hematoma que comprime y deforma la cabeza, dentro del cuerno derecho del útero bicorne. La flecha señala el cuerno uterino izquierdo.

cual producía compresión y deformidad de la cabeza fetal (figura 1). Se obtuvo un feto muerto de sexo masculino con varias deformidades macroscópicas (ausencia del hueso occipital, del fémur derecho e hiperflexión de la espina), restricción del crecimiento intrauterino, escasa cantidad de líquido amniótico y peso de 1950 gramos. Según el puntaje de Capurro, se estableció una edad gestacional de 37 semanas (figura 2). La placenta estaba fuertemente adherida a la pared uterina posterior y fue imposible separarla del sitio de implantación (figura 3).

En la exploración posterior se observó el cuerno derecho rudimentario no comunicante en un útero bicorne, con solución de continuidad que se extendía desde la unión hasta el fondo del cuerno rudimentario. La comunicación de este cuerno estaba cerrada por tejido conectivo fibroso. El cuerno izquierdo se comunicaba con un cuello uterino único. Las trompas de Falopio y los ovarios, de aspecto normal, se conectaban a 


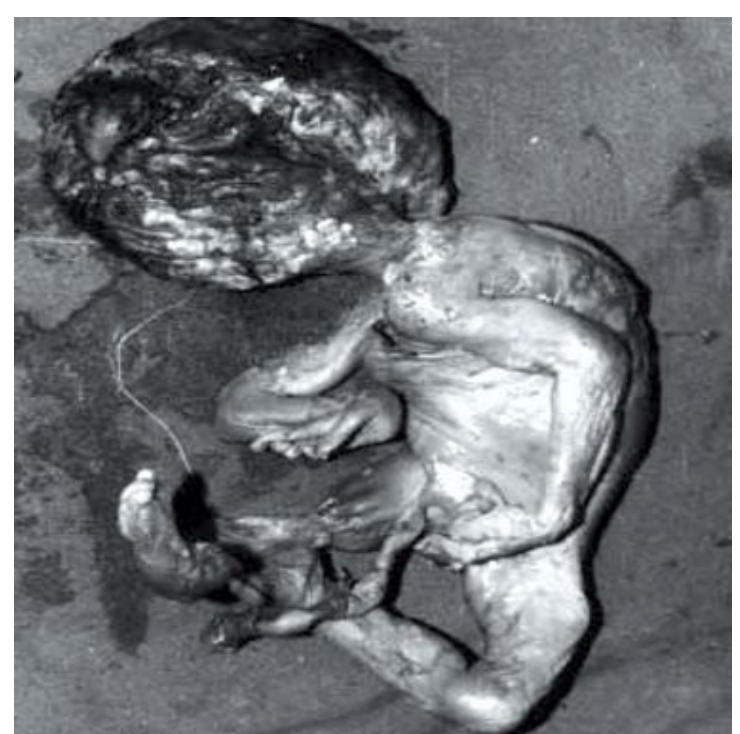

Figura 2. Feto con múltiples alteraciones congénitas: ausencia del hueso occipital, del fémur derecho e hiperflexión de la espina.

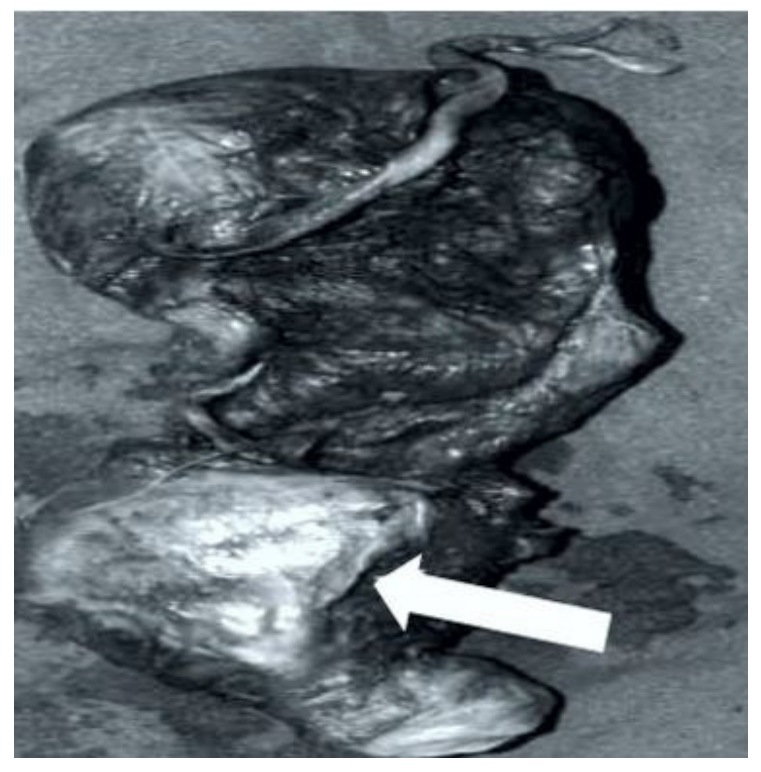

Figura 3. Cuerno derecho aumentado de tamaño con placenta y cordón umbilical en su interior, fuertemente adherida a la pared uterina posterior. La flecha señala el cuerno uterino derecho. cada cuerno del útero bicorne. Se realizó extracción del saco gestacional, feto y placenta e histerectomía subtotal con conservación del anexo izquierdo.

La evaluación de los riñones no demostró anormalidades macroscópicas. El examen histológico del saco gestacional confirmó el diagnóstico de cuerno rudimentario no comunicante con presencia de miometrio y evidencia de placenta ácreta. El periodo posoperatorio transcurrió sin incidentes y la paciente fue dada de alta a los 6 días.

\section{DISCUSIÓN}

Las malformaciones del conducto Mülleriano representan una variedad de anomalías congénitas producto de las alteraciones en el desarrollo, formación anormal o fusión incompleta de los conductos mesonéfricos (8). Estas condiciones se han relacionado con tasas elevadas de infertilidad, abortos recurrentes, prematuridad y otras complicaciones obstétricas, aunque en muchas pacientes pueden pasar desapercibidas (9-11). La verdadera incidencia de estas afecciones sigue siendo desconocida (10).

La rotura uterina acompañada de hemoperitoneo masivo durante el cuarto o quinto mes del embarazo es la presentación más común, se presenta en más del $90 \%$ de los casos $(1,5)$, mientras que alrededor del $10 \%$ de los embarazos llega al término (1). El grosor miometrial y el suministro vascular son suficientes para mantener al feto durante el primer trimestre del embarazo. Las deficiencias de la musculatura, la aparición de contracciones miometriales, un espacio intrauterino inadecuado y la vascularización ineficiente, junto con el aumento de la probabilidad de desarrollo acretismo placentario, son responsables de la rotura uterina en la mayoría de los casos (5). Este caso es inusual, ya que el embarazo llegó hasta el término sin rotura del cuerno uterino rudimentario. 


\section{EMBARAZO A TÉRMINO EN ÚTERO BICORNE CON CUERNO RUDIMENTARIO NO COMUNICANTE. REPORTE DE UN CASO}

Para realizar el diagnóstico pregestacional, generalmente se necesita la realización de histerosalpingografía, histeroscopia y laparoscopia, mientras que el diagnóstico prenatal se realiza con ecografía transvaginal (12). El diagnóstico preoperatorio es un desafío y menos del $5 \%$ de los casos reportados han sido diagnosticados durante el periodo preoperatorio (1-3). Los antecedentes obstétricos, como partos pretérmino previos o la interrupción del embarazo antes de las 20 semanas de gestación y/o la presencia de una tumoración de partes blandas asociadas a útero gestante en la ecografía, son claves para el diagnóstico preoperatorio (5). Se necesita un alto índice de sospecha sobre la base de los antecedentes obstétricos, junto con los hallazgos de ecografía, tomografía computada y resonancia magnética, para realizar el diagnóstico precoz del cuerno uterino rudimentario, permitiendo un manejo rápido y apropiado. Este tipo de abordaje no solo evita la mortalidad materno-fetal debida a rotura uterina, sino que también permite preservar la fertilidad futura de las pacientes.

La urografía con contraste, para excluir anomalías congénitas del tracto urinario, es obligatoria en todos los casos (3). Una vez que se diagnostica esta condición, el tratamiento apropiado es la escisión del cuerno uterino rudimentario, lo cual no interfiere con la capacidad reproductiva posterior (8). Aunque rara vez se necesita realizar histerectomía, la multiparidad y la edad avanzada fueron los factores decisivos para realizar la histerectomía en este caso.

El embarazo dentro del cuerno rudimentario no comunicante en el útero bicorne puede ocurrir por migración transperitoneal de los espermatozoides o del óvulo fertilizado desde las estructuras contralaterales (4). El cuerpo lúteo generalmente está del mismo lado del cuerno rudimentario en el que se produce el embarazo en el $90 \%$ de los casos (1). Sin embargo, en este caso fue imposible encontrar la ubicación del cuerpo lúteo debido a la edad gestacional.
El pronóstico del embarazo en el cuerno uterino rudimentario es generalmente malo. El miometrio del cuerno uterino rudimentario es muy delgado $\mathrm{y}$ el endometrio generalmente no es funcional, lo que se traduce, en la mayoría de los casos, en una la placentación patológica (14). Siempre es una emergencia cuando se produce la rotura del cuerno rudimentario, lo que generalmente ocurre entre 10 - 15 semanas de gestación $(1,13)$. Por lo tanto, si se realiza el diagnóstico de embarazo en un cuerno uterino rudimentario, se debe considerar extirpar el cuerno afectado, aunque existan casos esporádicos en los que se puede conseguir la supervivencia perinatal (2).

Se ha reportado que la tasa de supervivencia perinatal en estos casos es solo del $2 \%$. La alta mortalidad fetal ( $80 \%$ - $90 \%$ ) en estos casos se debe a la rotura uterina (3). Sin embargo, la evidencia de oligohidramnios severo, restricción del crecimiento intrauterino, anomalías congénitas y alteraciones placentarias fueron responsables de la muerte fetal en este caso. Las anomalías congénitas observadas en el presente caso podrían deberse a la limitación del espacio intrauterino, asociado al oligohidramnios (efecto de presión), que pudo interferir en forma importante con el desarrollo fetal adecuado.

\section{REFERENCIAS}

1. Siwatch S, Mehra R, Pandher DK, Huria A. Rudimentary horn pregnancy: a 10-year experience and review of literature. Arch Gynecol Obstet. 2013; 287(4):687-695. doi: 10.1007/s00404-012-2625-7.

2. Nahum GG. Rudimentary uterine horn pregnancy. A case report on surviving twins delivered eight days apart. J Reprod Med. 1997; 42(8):525-532.

3. Siristatidis C, Chrelias C, Kassanos D. Rudimentaryhorn pregnancy: some points to review. Arch Gynecol Obstet. 2011; 283(4):917-918. doi: 10.1007/s00404010-1658-z.

4. Mishra N, Yadav N, Koshiya D, Jhanwar V. Ruptured rudimentary horn pregnancy with a history of an uneventful vaginal delivery. J Med Ultrason. 2015; 42(1):117-120. doi: 10.1007/s10396-014-0582-4. 
5. Feteh VF, Dimala CA, Njim T, Fuka B. Post term pregnancy in a non-communicating rudimentary horn of a unicornuate uterus. BMC Res Notes. 2016; 9:209. doi: 10.1186/s13104-016-2013-7.

6. Bello J, Bruzual A, Rodríguez L. Útero bicorne y agenesia renal, asociado a preeclampsia: a propósito de un caso. Rev Fac Med (Caracas) [Internet]. 2012 [consultado julio de 2018]; 35(1):52-54. Disponible en: http://saber.ucv.ve/ojs/index.php/rev_fmed/article/ view/4920

7. Santimone M, Blanco R, Ledezma K, Rodríguez K, Sánchez M. Diagnóstico y tratamiento de un caso de malformación uterina. Centro Méd. 2001; 46(2): 134 137.

8. Caserta D, Mallozzi M, Meldolesi C, Bianchi P, Moscarini M. Pregnancy in a unicornuate uterus: a case report. J Med Case Rep. 2014; 8:130. doi: 10.1186/17521947-8-130.

9. Bhagavath B, Ellie G, Griffiths KM, Winter T, AlurGupta S, Richardson C, et al. Uterine Malformations: An Update of Diagnosis, Management, and Outcomes. Obstet Gynecol Surv. 2017; 72(6):377-392. doi: 10.1097/OGX.0000000000000444.

10. Vaz SA, Dotters-Katz SK, Kuller JA. Diagnosis and Management of Congenital Uterine Anomalies in Pregnancy. Obstet Gynecol Surv. 2017; 72(3):194-201. doi: 10.1097/OGX.0000000000000408.
11. Rackow BW, Arici A. Reproductive performance of women with müllerian anomalies. Curr Opin Obstet Gynecol. 2007; 19(3):229-237. doi: 10.1097/ GCO.0b013e32814b0649.

12. Juneja SK, Gupta S, Tandon P, Gumber N. Rupture of Noncommunicating Rudimentary Horn of Uterus. Int J Appl Basic Med Res. 2017; 7(2):146-147. doi: 10.4103/ ijabmr.IJABMR_112_16.

13. Huang YT, Yim SL, Kapurubandara S, Anpalagan A. Antepartum uterine rupture at 29 weeks gestation following unilateral salpingectomy and review of literature. BMJ Case Rep. 2017; 2017:bcr2015211359. doi: 10.1136/bcr-2015-211359.

14. Goluda M, St Gabryś M, Ujec M, Jedryka M, Goluda C. Bicornuate rudimentary uterine horns with functioning endometrium and complete cervical-vaginal agenesis coexisting with ovarian endometriosis: a case report. Fertil Steril. 2006; 86(2):462.e9-11. doi: 10.1016/j. fertnstert.2005.12.064.
Recibido 23 de agosto de 2020 Aprobado 18 noviembre de 2020 